\title{
Computational Prediction of Acoustic Waves from a Subscale Rocket Motor
}

\author{
By Taku NonOmurA ${ }^{1)}$, Seiichiro MorizawA ${ }^{2)}$, Shigeru OBAYASHI ${ }^{2)}$ and Kozo FuJII ${ }^{1)}$ \\ ${ }^{1)}$ Institute of Space and Astronautical Science, JAXA, Sagamihara, Japan \\ ${ }^{2)}$ Tohoku University, Sendai, Japan
}

(Received June 24th, 2013)

\begin{abstract}
For improvement of prediction of acoustics waves from a rocket plume, validation studies of numerical simulation are performed using experimental data of acoustics waves from subscale rocket motors, and prediction accuracy of numerical simulation is discussed. Experimental data of flow and acoustics fields of a solid motor and a H2-AIR liquid motor are used as the reference. The computational results of the far-field OASPL agree with experimental data within the error of approximately $5 \mathrm{~dB}$. The result shows that the current numerical methods well predict Mach waves at downstream side even though the results of far-field PSD are slightly overestimated. Meanwhile, it is difficult to capture shock associated acoustic waves and fine-scale turbulence acoustic waves at upstream side and the results of far-field PSD are underestimated. In addition, difference between single and multicomponent in the computational results is also small.
\end{abstract}

Key Words: Rocket Plume, Supersonic Jet, Computational Aeroacoustics, Multicomponent Compressible Flows

\section{Nomenclature}

$\begin{array}{lll}a & : & \text { sound of speed } \\ D & : & \text { nozzle diameter } \\ f & : & \text { frequency } \\ L & : & \text { distance from nozzle to the plate } \\ M & : & \text { Mach number } \\ p & : & \text { pressure } \\ \widetilde{p} & : \text { effective pressure fluctuation } \\ r & : \text { radial direction from jet axis } \\ R e & : \text { Reynolds number } \\ S P L & : \text { sound pressure level } \\ S t & : \text { Strouhal number } \\ T & : \text { temperature } \\ T_{\text {ratio }} & : \text { ratio of temperature } \\ u & : & x \text {-direction velocity } \\ \gamma & : & \text { specific heat ratio } \\ \mu & : & \text { coefficient of viscosity } \\ \rho & : & \text { density } \\ \theta & : & \text { angle axis from the jet axis }\end{array}$

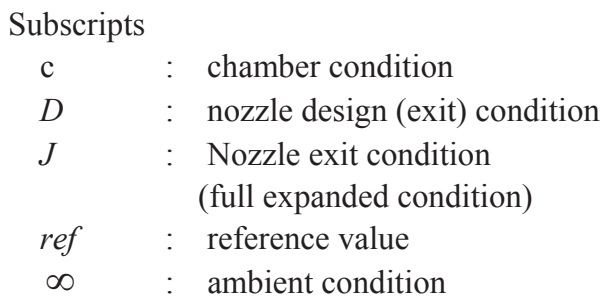

\section{Introduction}

It is well known that the rocket plume of a hot supersonic jet condition generates very strong acoustic waves which might be harmful to the payload inside the fairing of the rocket. Thus far, the empirical prediction model which is based on the database of free jets has been adopted to predict the intensity of acoustic waves. ${ }^{1,2)}$ Because of the empiricism of the prediction model, its prediction accuracy is significantly limited and it is difficult to quantitatively predict the intensity of acoustic waves, especially in the new launch site. Thus, a more accurate prediction model which is based on the physical model of acoustic waves is preferred. For this reason, it is necessary to understand the physical mechanism of acoustic wave generation.

The acoustic waves generated by a supersonic jet can be decomposed as shown in Fig. 1, ${ }^{3)}$ and the strong component is "acoustic waves from large-scale turbulent structure," which is so-called "Mach wave." In this study, the acoustic waves from the large-scale turbulent structure is focused on.

Recently, high fidelity numerical simulations, i.e. large-eddy simulations or direct numerical simulations, are becoming to accurately capture the acoustic waves from a supersonic jet. ${ }^{4-7)}$ This kind of numerical simulations seems to be useful to understand the physical mechanism of generation of the acoustic waves, because the numerical simulation provides the high resolution unsteady data of flow fields. ${ }^{8)}$

However, the reliability of the numerical simulation for the acoustic waves from a supersonic hot jet, like a rocket plume, has not been well assessed. Therefore, in this study, the validation study of the numerical simulation is conducted using solid and H2-AIR liquid motors. In addition, in this paper, the effects of modeling of the rocket plume, i.e. single gas-component or multiple(two)-gas-component modeling, and effects of reflection at the wall are discussed based on the results. 


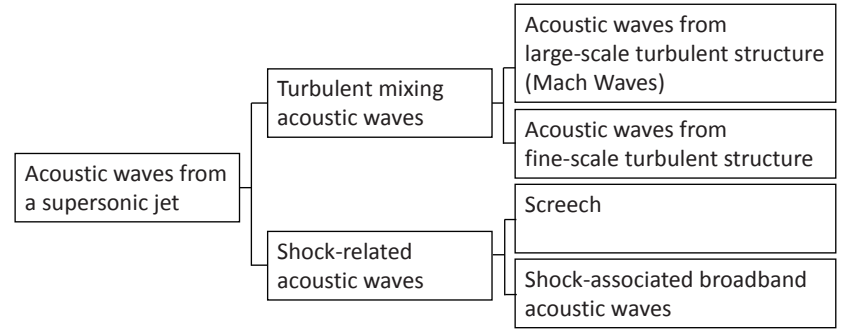

Fig. 1. Categorized acoustic waves from a supersonic jet.

\section{Experiment Data}

The experimental data of the liquid and solid rocket motors used in this paper is briefly presented in this section.

\subsection{Liquid rocket motor}

The specification of the liquid rocket motor is described in this subsection. Geometric parameters are as follows: the nozzle diameter $D$ is set to be $60[\mathrm{~mm}]$, and the distance from ground to the nozzle $L$ is set to be $3[\mathrm{~m}]$. The jet conditions are as follows: the exit jet velocity of the jet is $1,800[\mathrm{~m} / \mathrm{s}]$, the exit pressure is $3[\mathrm{MPa}]$, and the massflow rate is $1190[\mathrm{~g} / \mathrm{s}]$. The molecular weights and the specific heat ratio of the working fluid of the jet is 29.0 and 1.3 , respectively.

The microphones are located at $r=50 \mathrm{D}$ from the nozzle exit, as shown in Fig. 2. In this measurement, totally 11 microphones are employed for data acquisition. The angle of each microphone is shown in Table 1.

The distance from nozzle to the ground is long enough for the flow field and a free jet analysis seems to be good approximation for flow fields. Additionally the acoustic waves are not significantly reflected at the ground because we set the absorber at the ground. Therefore, we do not consider the acoustic wave reflection at the ground in this case.

Table 1. Microphone position for a liquid rocket motor.

\begin{tabular}{|l|l|l|l|l|l|l|l|l|l|l|l|}
\hline Mic. & M1 & M2 & M3 & M4 & M5 & M6 & M7 & M8 & M9 & M10 & M11 \\
\hline $\begin{array}{l}\text { Angle } \\
{[\mathrm{deg}]}\end{array}$ & 30 & 40 & 50 & 60 & 70 & 80 & 90 & 100 & 11 & 120 & 130 \\
\hline
\end{tabular}

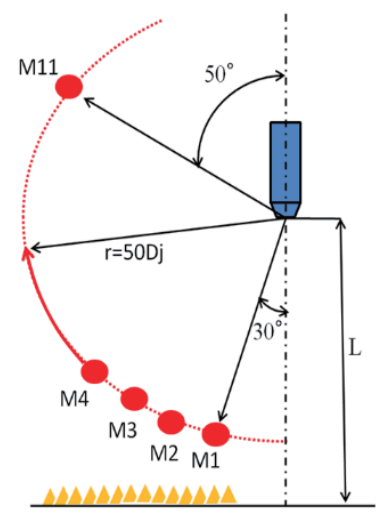

Fig. 2. Measurement configuration for a liquid rocket motor.

\subsection{Solid rocket motor}

The specification of the solid rocket motor is described in this subsection. Geometric parameters are as follows: nozzle the diameter $D$ is set to be $42.9[\mathrm{~mm}]$, and the distance from ground to the nozzle $L$ is set to be $2[\mathrm{~m}]$. The jet conditions are as follows: the exit jet velocity of the jet is $2,300[\mathrm{~m} / \mathrm{s}]$, the exit pressure is $9[\mathrm{MPa}]$, and the massflow rate is $450[\mathrm{~g} / \mathrm{s}]$. The molecular weights and the specific heat ratio of the working fluid of the jet is 26.4 and 1.17 , respectively.

The microphones are located at $r=75 \mathrm{D}$ from the intersection point of the jet axis and the ground, as shown in Fig. 3. In this measurement, totally 10 microphones are employed for data acquisition. The angle of each microphone is shown in Table 2.

The distance from nozzle to the ground is long enough for the flow field and a free jet analysis seems to be good approximation for flow fields, also in this case. However, the acoustic waves are significantly reflected at the ground because no absorber is used at the ground. Therefore, we consider the acoustic wave reflection effects as discussed later.

Table 2. Microphone position for a solid rocket motor.

\begin{tabular}{|l|l|l|l|l|l|l|l|l|l|l|}
\hline Mic. & M1 & M2 & M3 & M4 & M5 & M6 & M7 & M8 & M9 & M10 \\
\hline $\begin{array}{c}\text { Angle } \\
{[\mathrm{deg}]}\end{array}$ & 8 & 17.7 & 25.9 & 34.9 & 43.4 & 52.4 & 60.7 & 69.9 & 74 & 84.3 \\
\hline
\end{tabular}

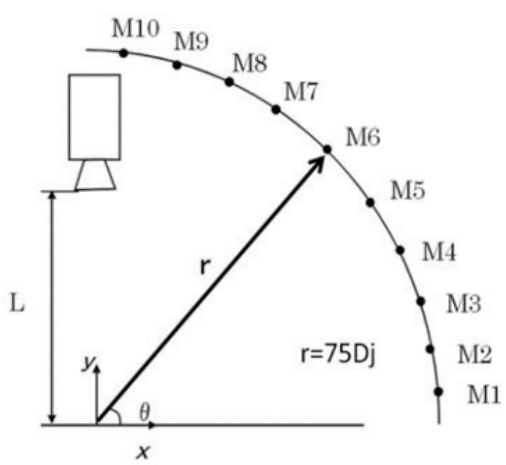

Fig. 3. Measurement configuration for a solid rocket motor.

\section{Parameters of Jets, and Modeled Flow Condition}

Air and mixed jet gases are used for the working fluid in the present analysis. The two type of simulations are conducted: one is computation with the single-component-gas modeling in which the ambient gas is assumed to be the same as the jet gas, and the other is computation with the multi-component-gas modeling in which the ambient gas is assumed to be air.

Four jet parameters are employed to describe the jet condition: the ideally expanded Mach number, the nozzle exit Mach number, the Reynolds number, and the temperature ratio.

The ideally expanded Mach number is determined only by the ratio of pressures at the chamber and ambient, as follows:

$$
M_{J}=\frac{u_{J}}{a_{J}}=\sqrt{\frac{2}{\gamma-1}\left(\left(\frac{p_{c}}{p_{\infty}}\right)^{\frac{\gamma-1}{\gamma}}-1\right)}
$$

Here, subscripts $J, \infty, c$ denote the ideally expanded condition, ambient condition, and the chamber condition, respectively, and $u, p, a, \gamma$ are the velocity, the pressure, the speed of sound, and the specific heat ratio. 
In addition, $M_{D}, T_{\text {ratio }}, R e$, are defined as follows:

$$
\begin{gathered}
M_{D}=\frac{u_{D}}{a_{D}} \\
T_{\text {ratio }}=\frac{T_{j}}{T_{\infty}} \\
\operatorname{Re}=\frac{\rho_{D} u_{D} D}{\mu_{D}}
\end{gathered}
$$

Here, $\rho, \mu, T, D$, are the density, the viscosity, the temperature, and the nozzle diameter, respectively. In this study, the Reynolds number is set to $10^{5}$ which is lower than the actual value, to reduce the computational costs. Insensitivity of the Reynolds number on the qualitative characteristics of the Mach wave emission has been well investigated. Note that, if we compute with the actual Reynolds number, such as $10^{6}$ to $10^{8}$, required computational resources becomes 100 to 1000 times higher than the present computation, and it seems to be almost impossible with the present computational environments.

The jet parameters of the liquid and solid rocket motors are shown in Tables 3 and 4, respectively. The jet profile at the nozzle exit is shown in Fig. 4. Here, $r$ denotes the distance from the center of the nozzle. In this study, the very thin shear-layer (on the 4 computational points) is employed, which promotes the instability of the shear layer. Therefore, any artificial forcing on the shear layer is not applied.

The sound pressure level and Strouhal number are defined as follows:

$$
\begin{gathered}
S t=\frac{f D}{u_{D}} \\
S P L=20 \log _{10} \frac{\tilde{p}}{p_{r e f}}
\end{gathered}
$$

Here, $\tilde{p}$ is the effective pressure fluctuation, and $p_{\text {ref }}$ is the minimum audible sound pressure defined as follows:

$$
p_{\text {ref }}=\frac{2.0 \times 10^{-5}[\mathrm{~Pa}]}{101300[\mathrm{~Pa}]} \times p_{\infty}
$$

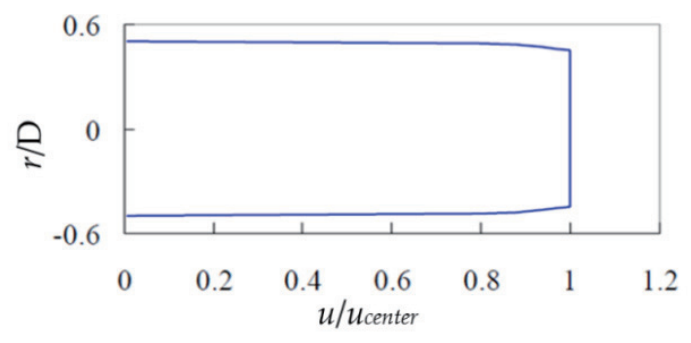

Fig. 4. Jet profile.

Table 3. Jet parameters of a liquid rocket motor.

\begin{tabular}{cccccccc}
\hline \hline Type & $\boldsymbol{M}_{\boldsymbol{J}}$ & $\boldsymbol{M}_{\boldsymbol{D}}$ & $\boldsymbol{p}_{\boldsymbol{D}} / \boldsymbol{p}_{\infty}$ & $\boldsymbol{T}_{\text {ratio }}$ & $\boldsymbol{U}_{\boldsymbol{D}} / \boldsymbol{a}_{\infty}$ & $\boldsymbol{\gamma}_{\text {jet }}$ & $\boldsymbol{\gamma}_{\infty}$ \\
\hline Multi & 2.79 & 3.10 & 0.60 & 6.48 & 5.25 & 1.3 & 1.4 \\
Single & 2.79 & 3.10 & 0.60 & 6.48 & 5.13 & 1.3 & 1.3 \\
\hline \hline
\end{tabular}

Table 4. Jet parameters of a solid rocket motor.

\begin{tabular}{lccccccc}
\hline \hline Type & $\boldsymbol{M}_{\boldsymbol{J}}$ & $\boldsymbol{M}_{\boldsymbol{D}}$ & $\boldsymbol{p}_{\boldsymbol{D}} / \boldsymbol{p}_{\infty}$ & $\boldsymbol{T}_{\text {ratio }}$ & $\boldsymbol{U}_{\boldsymbol{D}} / \boldsymbol{a}_{\infty}$ & $\boldsymbol{\gamma}_{\boldsymbol{j e t}}$ & $\boldsymbol{\gamma}_{\infty}$ \\
\hline Multi & 3.27 & 3.63 & 0.48 & 12.3 & 8.99 & 1.17 & 1.4 \\
Single & 3.27 & 3.63 & 0.48 & 12.3 & 9.49 & 1.17 & 1.17 \\
\hline \hline
\end{tabular}

\section{Numerical Methods}

The large-eddy simulation is conducted to predict the acoustic waves from the rocket plume. As discussed before, the free jet is assumed for the fluid analysis because the ground is far enough for the jet flow. In the far-field acoustic wave prediction using a Kirchhoff method, the acoustic wave reflection is not considered in the case of a liquid rocket motor because absorber is used in the experiment, while it is considered when the far-field acoustic waves are predicted by Kirchhoff method in the case of a solid rocket motor.

With regard to the fluid analysis, following numerical methods are employed. The governing equations are the three-dimensional Navier-Stokes equations, which is normalized by the density of the ambient condition, the sound speed of ambient condition and the diameter of the exit nozzle, and mass-fraction equation. These equations are solved in the curvilinear coordinate system. The modified weighted compact nonlinear scheme (WCNS) ${ }^{9-11)}$ is employed for discretization of advection terms. In the flux evaluation procedure of WCNS, simple high-resolution upwind scheme (SHUS) ${ }^{12)}$ is employed. A sixth-order central difference scheme is employed for discretization of viscous terms. With regard to the time integration, a second order backward difference scheme which is converged by the alternate-directional implicit symmetric Gauss-Seidel (ADI-SGS) scheme ${ }^{13)}$ is employed. In the case of computation of multiple-gas-component modeling, the mass-fraction equation is solved with the conservative form which generates spurious numerical errors, but we try to minimize the error using the primitive variable interpolation. ${ }^{14)}$ In this framework, we can conserve the mass of different species with minimum error. With regard to the turbulence modeling, the explicit subgrid model is not used, thus the modeling of the present computation is categorized into monotonically integrated large eddy simulation (MILES). ${ }^{15)}$

For the sound pressure evaluation, the pressure fluctuation is directly sampled as sound pressure inside the resolved region, and it is evaluated by the frequency domain Kirchhoff method based on the surface pressure data at the outside boundary of the resolved region. ${ }^{16)}$ The acoustic waves from a free jet is simply considered for the liquid rocket motor, while reflection waves from the ground is considered for the solid rocket motor. With regards to the solid rocket motor, a mirror image of Kirchhoff integral surface under the ground is also considered and integration is conducted over real and mirrored Kirchhoff surfaces to include the effects of reflection waves, as shown in Fig. 5. This assumption is corresponding to the perfect reflection at the ground. In this paper, the effects of reflection waves are included in this way.

Figure 6 shows the computational region. The computational region consists of a resolved region and a buffer region. Flow and acoustic waves are resolved by the computational scheme in the resolved region, and they are intentionally dumped by the stretched grid in the buffer region. ${ }^{17)}$

Figure 7 shows the computational grid. The total number of 
grid points is approximately 10 million points. The grid convergence study is also conducted with the grid of 30 million points, and the difference in overall SPL (OASPL) distribution of the fine and coarse grids is found to be less than $2 \mathrm{~dB}$. In the present paper, only the results with the grid of 10 million points are shown.

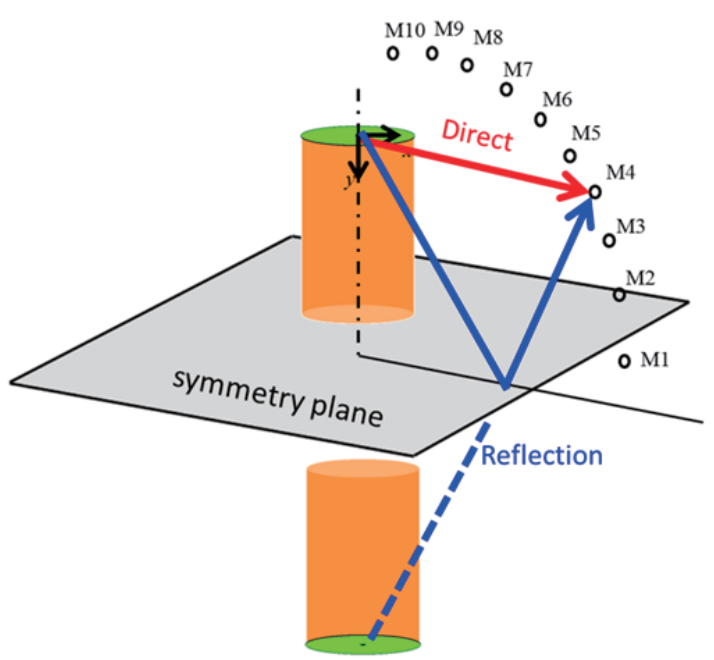

Fig. 5. Image of reflection waves in Kirchoff method.

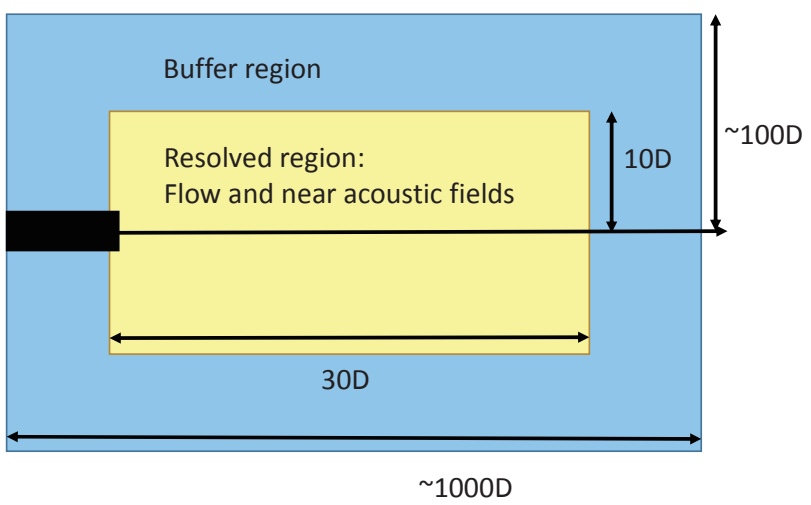

Fig. 6. Computational region.

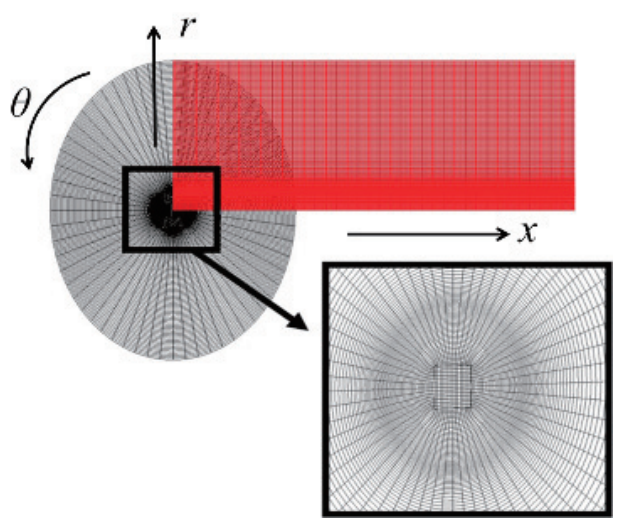

Fig. 7. Computational grid.

\section{Results}

\subsection{Results of the liquid rocket motor}

In this section, the experimental and computational data of the liquid rocket motor are compared and discussed. As noted before, two types of computations are conducted: the computation with single-gas-component modeling in which the ambient gas is the same as the jet gas, and the computation with the multiple-gas-component modeling in which the ambient gas is different from the jet gas.

Figure 8 shows the instantaneous flow fields obtained by computation. Figure 8 clearly illustrates the existence of the Mach waves in both cases. Moreover, the relevant reflection is not observed in the boundary of resolved region (which corresponds to the boundary of Fig. 8), which shows the buffer region suppresses the reflection as similar to the previous work. ${ }^{6,7)}$

Then, the directivity of OASPL is discussed. Figure 9 shows the directivity at the far-field. Computation predicts the sound pressure level within the error of $5[\mathrm{~dB}]$. The OASPL of computational results are higher than that of experimental data. This trend is clearly observed in low angle region in which the Mach wave emission might be dominant. This is possibly because the strength of thee Mach wave which is generated by the large scale structure is overestimated in the computation. This might be caused by the overestimation of turbulence structure due to the lack of the inflow disturbance, similar to the subsonic jet computed by Bogey et al. ${ }^{18)}$

Next, the frequency characteristics of the sound pressure at far fields are discussed. Figure 10 shows the power spectral densities (PSDs) of the sound pressure at far-fields. Here, Figs. 10(a) and 10(b) are PSDs at the downstream and upstream sides, respectively. The PSD at the downstream side is much affected by the Mach wave which is obliquely propagated to the downstream side, and the PSD at the upstream side is much affected by the fine scale turbulent noise which is omnidirectionally propagated and is clearly observed in the region where the Mach wave is not observed. ${ }^{19)}$ Using the data at the both location, the prediction accuracy for the PSD is discussed. Comparing the experimental and computational data, the computation well predicts the frequency characteristics qualitatively. More quantitatively, the SPL of the computation is overpredicted at the downstream side (Fig. 9(a)). This is because the Mach wave strength is overestimated in the computation as discussed before. Moreover, the SPL of the computation is underpredicted at the upstream side (Fig. 10(b)). This is because the fine scale turbulence are not resolved well due to the insufficient grid resolution.

Finally, the difference in single-gas and multiple-gas-component is discussed. Figures 9 and 10 show that the difference in the predicted SPL is less than $2[\mathrm{~dB}]$, and this clarifies that the effects of taking the difference of gas components into account on the directivity and the frequency characteristics are small. 


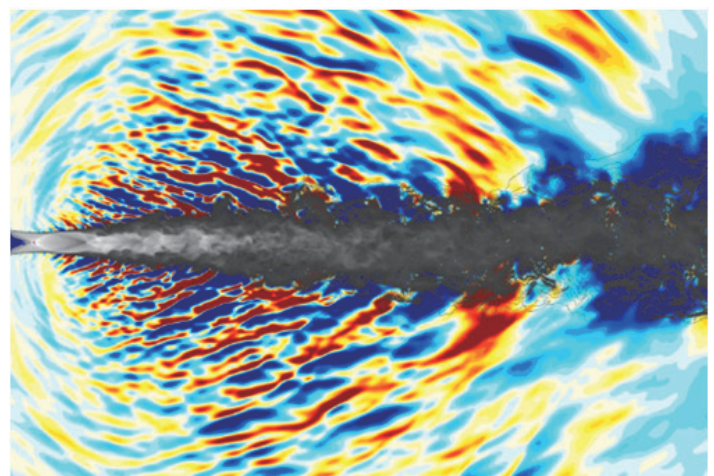

(a) Multi-component computation

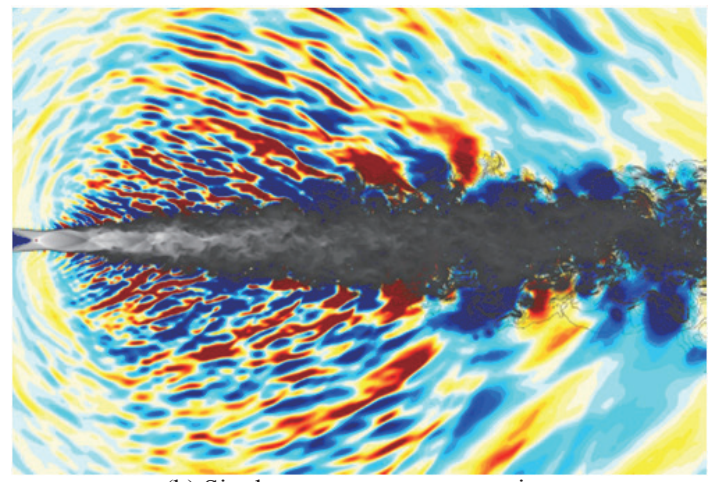

(b) Single-component computation

0.97 Statistic pressure 1.03

$0 \quad$ Dynamic pressure 8

Fig. 8. Instantaneous flow fields of liquid rocket motor.

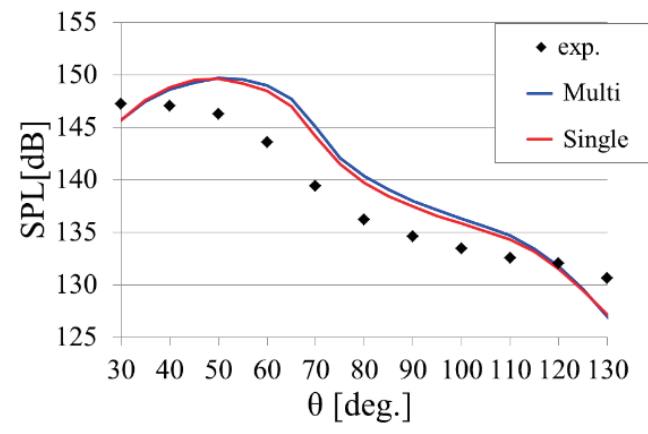

Fig. 9. OASPL of a liquid rocket motor at far-field.

\subsection{Results of the solid rocket motor}

In this Section, the experimental and computational data of the solid rocket motor are compared and discussed, similar to the liquid rocket motor. Also in this computation, two type of computation are conducted: the computations with the single-gas-component modeling and multiple-gas-component modeling.

Figure 11 shows the instantaneous flowfields obtained by computation. Figure 11 clearly illustrates the existence of Mach waves in both cases, where the Mach wave is much stronger than those of the liquid rocket motor. Again, the relevant reflection is not observed in the boundary, which shows the buffer region suppress the reflection well.

The directivity of OASPL is discussed. Figure 12 shows the directivity at the far-field. It should be noted that those OASPL

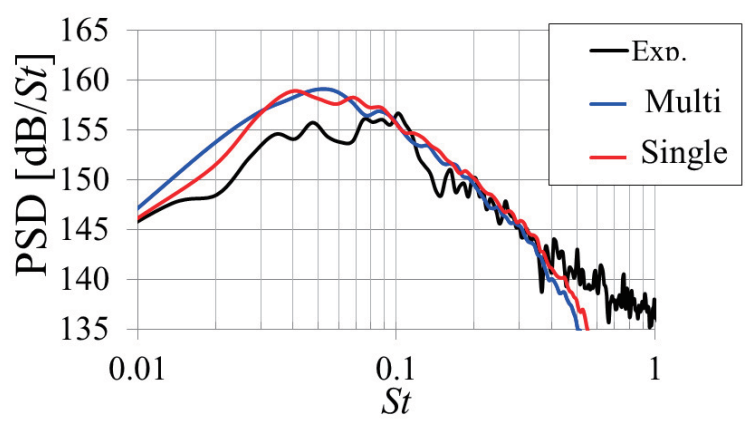

(a)PSD of SPL at downstream side (M2, 40[deg])

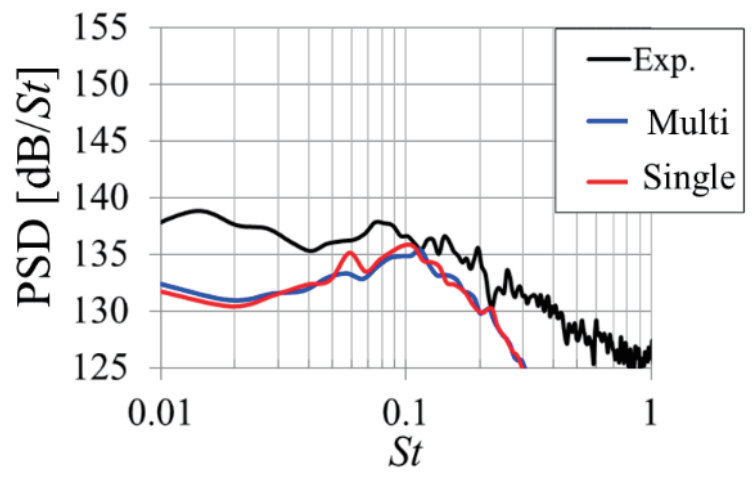

(b)PSD of SPL at downstream side (M11, 110[deg])

Fig. 10. PSD of SPL for a liquid rocket motor.

data includes the reflected acoustic waves by the ground. In the computation, this effects are taken into account when the Kirchhoff method is applied as discussed before. Here, the SPL of the computational results agree well with that of the experimental data with the maximum error of $5[\mathrm{~dB}]$. The frequency characteristics of the sound pressure at far fields are discussed. Figure 13 shows the power spectral densities (PSDs) of the sound pressure at far-fields. Here, Figs. 13(a) and 13(b) are PSDs at the downstream and upstream sides, respectively. Comparing the experimental and computational data, the computation well predicts the frequency characteristics within the error of $5[\mathrm{~dB}]$. The SPL of the computation is overpredicted at the downstream side (Fig. 13(a)). This is again because the Mach wave strength is overestimated in the computation as discussed before. The SPL of the computation is underpredicted at the upstream side (Fig. 13(b)). This is also because the fine scale turbulence are not resolved well due to insufficient grid resolution.

In this problem, data contains the reflection effect. This effect is decomposed. Figure 14 shows the far-field OASPLs computed by the direct wave only, by the reflected wave only, and by the both the direct and reflected waves, respectively. The reflection effects are strong at near the ground and the upstream regions. This result shows that the reflected wave takes a part at near the ground and upstream regions. It is important to consider the reflection wave at those region when the similar configuration is adopted.

In this case, the difference in single-gas and multiple-gas-component modeling is also investigated and the effects of gas components on the directivity and the frequency characteristics are found to be small. 


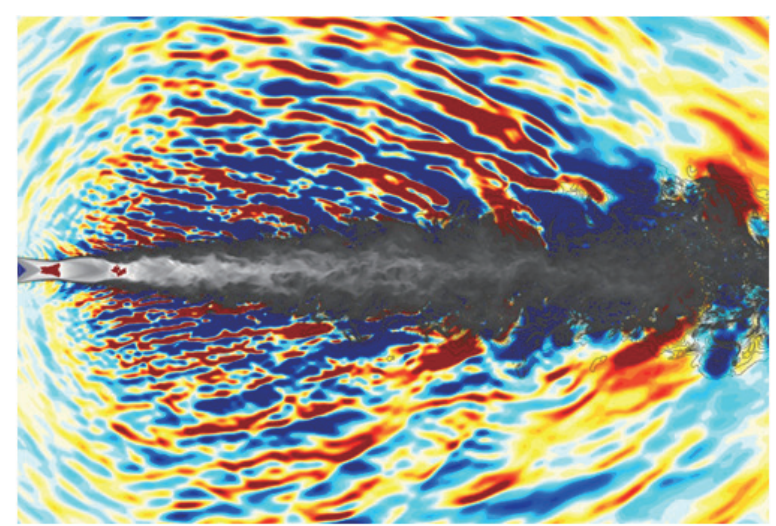

(a) Multi-component computation

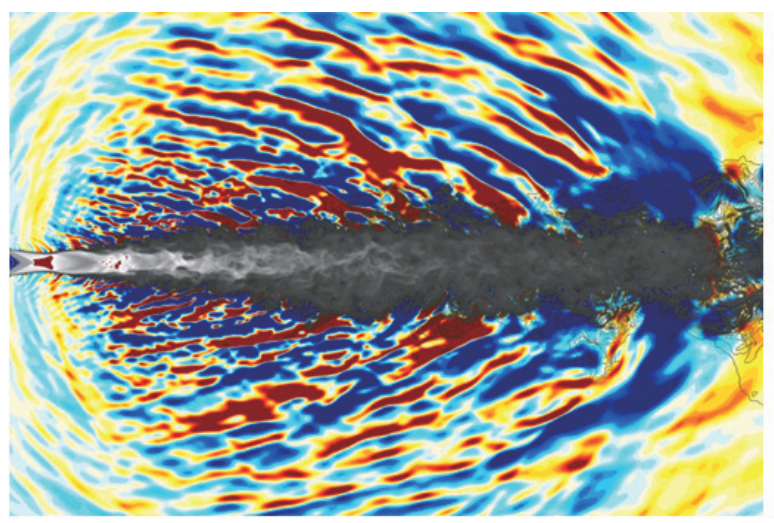

(b) Single-component computation

0.97 Statistic pressure 1.03

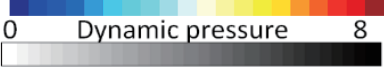

Fig. 11. Instantaneous flow fields of solid rocket motor.

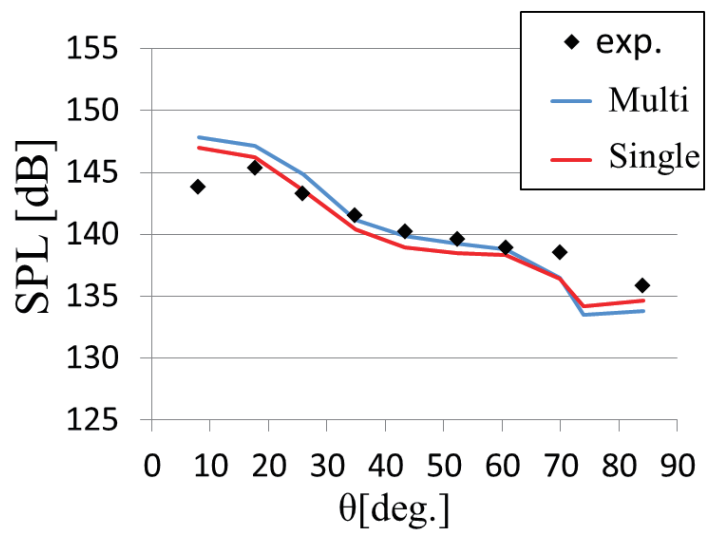

Fig. 12. OASPL of a solid rocket motor at far-field.

\section{Conclusions}

In the present study, the computational prediction of the acoustic waves from liquid and solid motors are compared with experimental data, and its reliability is discussed. In addition, the modelling of single-gas/multiple-gas-component gases and effects of reflection are also discussed in this framework.

As a result, the OASPL of the computational data agrees well with that of the experimental data within the $5 \mathrm{~dB}$ error, and the computational data can provide the qualitative data of the

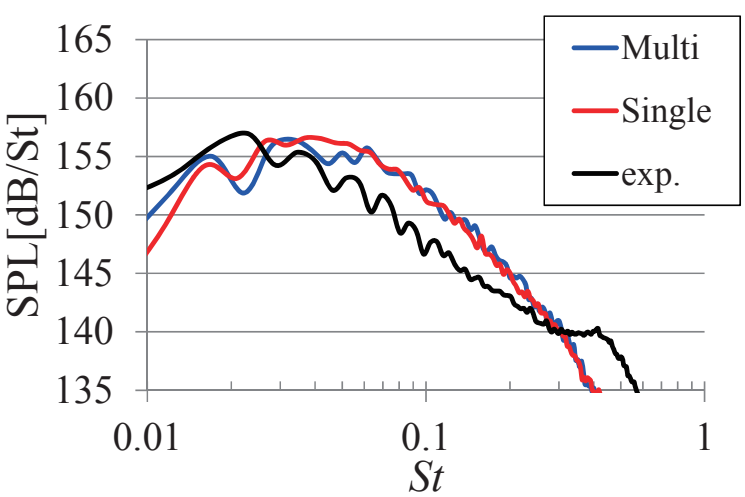

(a) PSD of SPL at downstream side (M1, 8[deg])

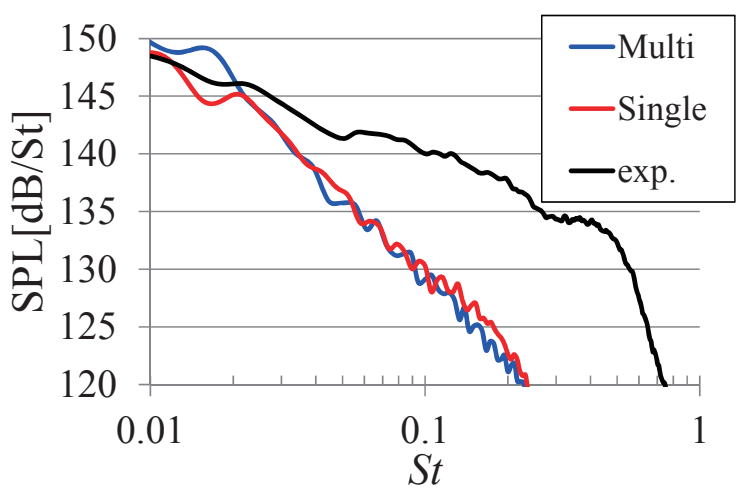

(b)PSD of SPL at upstream side (M10, 84.3[deg])

Fig. 13. PSD of SPL for a solid rocket motor.

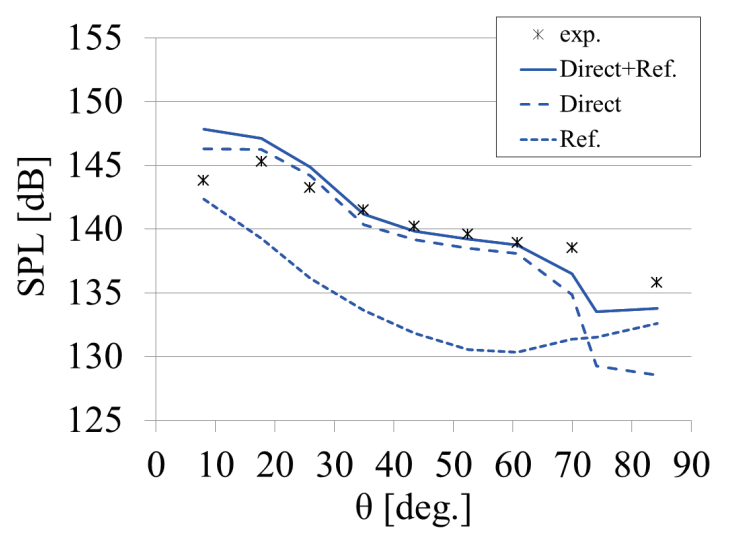

Fig. 14. Decomposition of OASPL into direct wave and reflection wave components.

frequency characteristics. The results also indicate that the computation can predict the Mach wave which is generated from the large scale structure of turbulence, while it is difficult to predict the acoustic wave at the upstream side which might be generated by the fine scale turbulent structure and the shock turbulent interaction.

Moreover, the present results illustrate that the modelling of gas component does not significantly affect the result, and that the reflection effect is significant at near the ground and upstream regions in the present condition of the experiment of the solid motor. 


\section{Acknowledgments}

The data obtained through JAXA/CNES collaboration works are used. The authors are grateful to Drs. Nobuhiro Yamanishi, Seiji Tsutsumi, Keita Terashima and collaborators in CNES side, who contributed to this collaboration work. The computation was conducted with JAXA super computer system. This research is partially supported by KAKENHI(25709009). The code used in this study was developed in the research supported by KAKENHI(23760773).

\section{References}

1) Eldred, S.: Acoustics Loads Generated by Propulsion System, NASA SP-8072, 1971.

2) Varnier, J., and Raguenet, W.: Experimental Characterization of the Sound Power Radiated by Impinging Supersonic Jets, AIAA Journal, 40 (2002), pp. 825-831.

3) Tam, C. K.: Jet Noise: Since 1952, Theoretical Computational Fluid Dynamics," 10(1998), pp. 393-405.

4) Bailly, C., and Bogey, C.: Contributions of Computational Aeroacoustics of Jet Noise Research and Prediction, International Journal of Computational Fluid Dynamics, 18 (2004), pp.481-491.

5) Bodony, D., and Lele, S.: Current Status of Jet Noise Prediction Using Large-Eddy Simulation, AIAA Journal, 46 (2008), pp. 364-380.

6) Nonomura, T. and Fujii, K.: Overexpansion Effects on Characteristics of Mach Waves from a Supersonic Cold Jet, AIAA Journal, 49 (2011), pp. 2282-2294.

7) Nonomura, T., Goto, Y. and Fujii, K.: Aeroacoustic Waves Generated from a Supersonic Jet Impinging on an Inclined Flat Plate, International Journal of Aeroacoustics, 10 (2011), pp. 401-426.

8) Fujii, K., Nonomura, T. and Tsutsumi: Toward accurate simulation and analysis of strong acoustic wave phenomena - A review from the experience of our study on rocket problems, International Journal of Numerical Methods in Fluids, 64 (2010) pp. 1412-1432.

9) Nonomura, T. and Fujii, K.: Effects of Difference Scheme Type in High-order Weighted Compact Nonlinear Schemes, Journal of Computational Physics, 228 (2009), pp. 3533-3539.

10) Nonomura, T., Iizuka, N. and Fujii, K.: Freestream and Vortex Preservation Properties of High-order WENO and WCNS on Curvilinear Grids, Computers \& Fluids, 39 (2010), pp. 197-214.

11) Nonomura, T., Li, W., Goto, Y. and Fujii, K.: Efficiency Improvements in Seventh-order Weighted Compact Nonlinear Scheme, CFD Journal, 18 (2011), pp. 180-186.

12) Shima, E., and Jounouch, T.: Role of CFD in Aeronautical Engieering (No14) -AUSM type Upwind Schemes -, Proceedings of the 14th NAL Symposium on Aircraft Computational Aerodynamics, 1997, pp. 7-12.

13) Nishida, H. and Nonomura, T.: ADI-SGS Scheme on Ideal Magnetohydrodynamics, Journal of Computational Physics, 228 (2008), pp. 3182-3188.

14) Nonomura, T., Morizawa, S., Terashima H. Obayashi S. and Fujii, K.: Numerical (Error) Issues on Compressible Multicomponent Flows Using a High-order Differencing Scheme: Weighted Compact Nonlinear Scheme, Journal of Computational Physics, 231 (2012), pp.3181-3210.

15) Boris, J. P., Grinstein, F. F., Oran, E., and Kolbe, R. J.: New Insights into Large Eddy Simulation, Fluid Dynamics Research, 10 (1992), pp.199-228.

16) Pan, F., I., Uzun, A., and Lyrintis, A., S.: Refraction Correction for Surface Integral Methods in Jet Aeroacoustics, AIAA paper 2004-2873, 2004.

17) Colonius, T., Lele, S. K., and Moin, P.: Boundary condition for Direct Computation of Aerodynamics Sound Generation, AIAA Journal, 31 (1993), pp. 1574-1582.
18) Bogey C., Marsden, O., and Bailly, C.: A Computational Study of the Effects of Nozzle-exit Turbulence Level on the Flow and Acoustic Fields of a Subsonic Jet, AIAA paper 2011-2837, 2011.

19) Tam, C. K., Viswanathan, K., Ahuja, K. K., and Panda, J., The Sources of Jet Noise: Experimental Evidence, AIAA 2007-3641, 2007. 